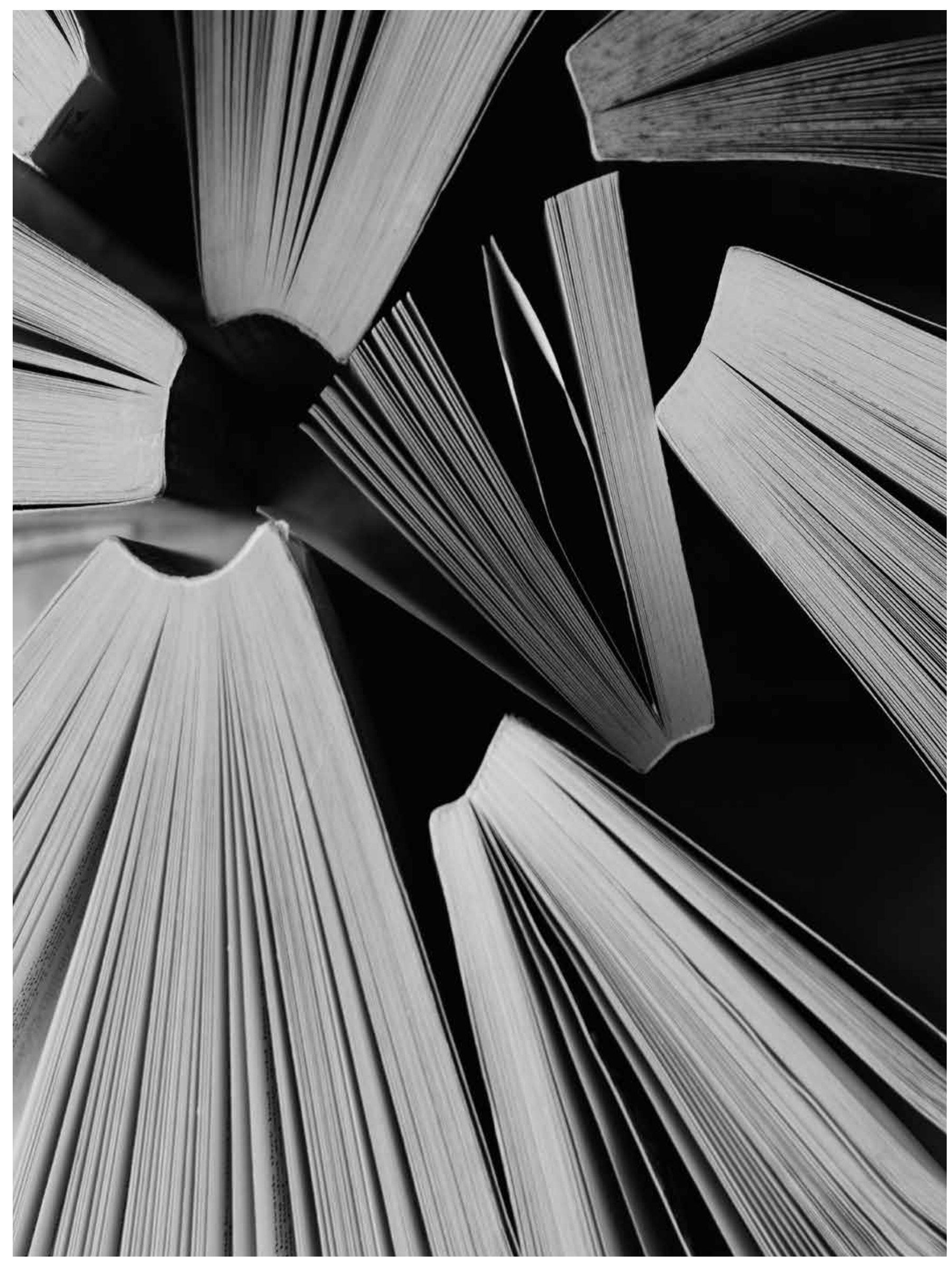




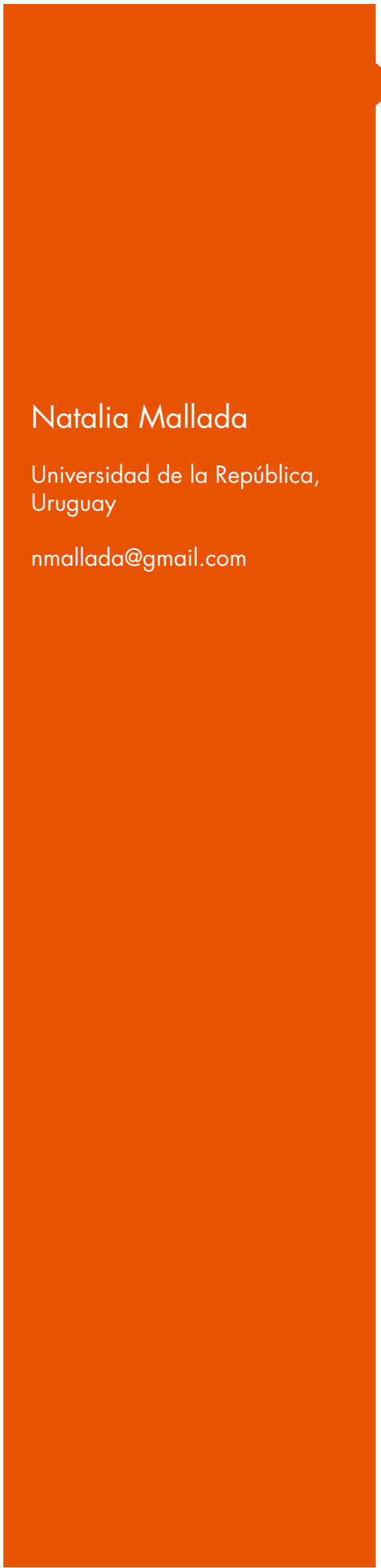

\section{Enseñar en las universidades y en los institutos de formación docente}

Compiladora: Mónica Insaurralde

Publicación de Noveduc

Buenos Aires, 2016

Esta obra compila una muestra de las ponencias que se presentaron en las II Jornadas Internacionales «Problemáticas en torno a la Enseñanza en la Educación Superior. Diálogo abierto entre la Didáctica General y las Didácticas Específicas». Dichas jornadas, realizadas en la Universidad de Luján en agosto de 2015, tuvieron como propósito propiciar un espacio de reflexión y debate sobre las problemáticas actuales y los desafíos de la enseñanza en este nivel educativo.

La publicación consta de un prólogo, a cargo de Mónica Insaurralde, y de cinco partes que se organizan en torno a las preocupaciones que atravesaron los diferentes ejes temáticos del evento. En el prólogo, Insaurralde realiza una contextualización con respecto a las Jornadas y adelanta los temas que se tratan en las diferentes partes del libro. En todos los casos, a pesar del carácter internacional de la actividad, los autores de las ponencias provienen de instituciones de educación superior argentinas.

La primera parte, denominada «Aprendizajes de docentes y estudiantes en las aulas universitarias. Experiencias e investigaciones», presenta cinco capítulos sobre innovaciones de diferente tipo que buscan mejorar la enseñanza y los aprendizajes. En ellos, se exponen y analizan diversas experiencias de enseñanza o de acompañamiento a los aprendizajes en diferentes carreras universitarias: el sistema de tutorías en una carrera de odontología, la articulación teoríapráctica en una carrera de nutrición, los modos en que aprenden a enseñar química los docentes universitarios, así como algunos aspectos acerca de la enseñanza de la enfermería y de anatomía e histología.

La segunda parte, «El ingreso a las universidades públicas: reflexiones sobre experiencias de enseñanza», «se ocupa — a juicio de Insaurralde - de uno de los desafios actuales de la educación superior: cómo colaborar en la configuración del oficio de estudiante universitario». ${ }^{1}$ En los tres capítulos de esta parte se exponen, respectivamente, aspectos sobre la enseñanza de la matemática en primer año de la universidad, una experiencia de alfabetización académica ${ }^{2}$ dirigida a estudiantes de la materia Química y una experiencia de diseño de materiales digitales para favorecer la comprensión lectora en carreras de ingeniería.

La tercera parte se dedica a «La enseñanza de la investigación en carreras de grado y posgrado». En tres capítulos, se abordan asuntos tales como el modo en que se enseña investigación en una carrera de ciencias sociales, aspectos de la formación en investigación dirigida a maestrandos y asuntos relativos a

1 Aquí, la conceptualización planteada por la autora parece diferir de la de Philippe Perrenoud, ya que para él este oficio implica estrategias que exceden el ámbito escolar y que escapan a las expectativas del mundo adulto.

2 Entendida esta en un sentido laxo, y no en el que propone Paula Carlino, relativo a la lectura y la escritura universitarias. 
Enseñar en las

universidades y en los

institutos de formación

docente

Mónica Insaurralde

(compiladora)

Publicación de Noveduc

(2016), Buenos Aires la enseñanza de didáctica general para estudiantes de profesorado de ciencias jurídicas.

La cuarta parte, «La formación de futuros profesores», se centra en las relaciones entre saber didáctico y práctica pedagógica en estudiantes de profesorado, en el potencial constructivo del cine en la formación de profesores y en la problematización del sentido común en psicología de la infancia, con estudiantes de formación docente.

La quinta parte aborda «La evaluación como estrategia metacognitiva en la educación superior». Allí se incluyen un capítulo sobre narrativas en la enseñanza de matemáticas y una experiencia en la que se asume la corrección como parte del proceso de enseñanza en un curso de didáctica.

Por último, en la sexta parte, «Experiencias y desafios pendientes en la enseñanza mediada por las TIC», se presenta un blog de escritura en la formación en didáctica de la lengua y literatura, el uso de TIC en la formación de profesores de música, aspectos sobre la construcción de ciudadanía en estudiantes virtuales de profesorado y reflexiones acerca del uso de TIC en la enseñanza universitaria a partir de la vivencia de estudiantes online.

En definitiva, se trata de un texto interesante para docentes universitarios y de formación docente preocupados por mejorar sus prácticas de enseñanza. También es un útil material de referencia para asesores pedagógicos y para quienes trabajan en temas de diseño curricular. Las experiencias y reflexiones presentadas refieren al acompañamiento a los estudiantes en el ingreso universitario, al tratamiento de diversos contenidos a enseñar, al empleo de diferentes recursos didácticos, al aprendizaje de la docencia, al abordaje de la lectura y la escritura como parte del proceso de enseñanza y a la incorporación de TIC en la enseñanza.

Tal vez, el hecho de que el lector acceda a las ponencias separadas del contexto original en que fueron enunciadas haga que se pierda parte de ese diálogo entre didáctica general y didácticas específicas que sugiere el título del evento. Es tarea del lector, con la ayuda del prólogo, la reconstrucción de esos puentes. Para continuar el recorrido, también es recomendable la lectura del libro La enseñanza en la educación superior. Investigaciones, experiencias y desafíos, compilado por la misma autora, en el que se comparten los aportes de los conferencistas y panelistas que participaron en las Jornadas. 


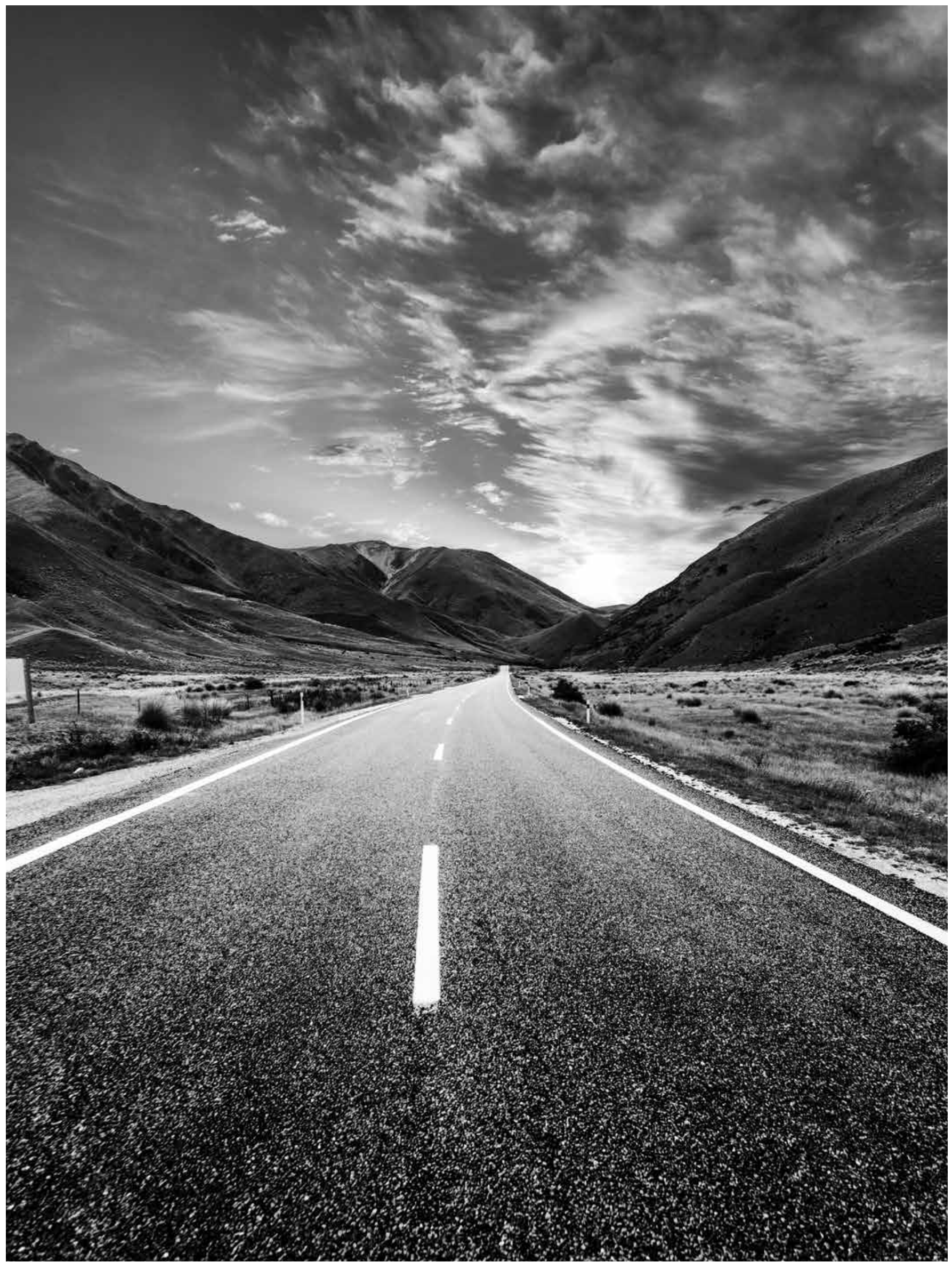

